\title{
Lecithin to Sphingomyelin Ratio Measurement
}

National Cancer Institute

\section{Source}

National Cancer Institute. Lecithin to Sphingomyelin Ratio Measurement. NCI Thesaurus.

Code C147381.

The determination of the ratio of lecithin compared to sphing omyelin present in a sample. The measurement may be expressed as a ratio or percentage. 